\title{
Stereotactically guided breast biopsy: a review
}

\author{
Victoria Ames • Peter D. Britton
}

Received: 27 August 2010 /Revised: 3 December 2010 / Accepted: 22 December 2010 / Published online: 20 January 2011

(C) European Society of Radiology 2011

\begin{abstract}
The aims of this review are to compare and contrast the available stereotactic equipment, and to describe the variety of needle types used and their affect on pathological results and subsequent patient management. Initial stereotactic devices were "added-on" to analogue mammography units and have been replaced by prone or ducubitus equipment using digital image acquisition. Biopsies use either 14-G core biopsy (CB) needles or vacuum-assisted biopsies (VAB). Vacuum-assisted biopsy systems consistently out-perform 14-G CB with reduced need for diagnostic or multi-treatment surgery. The falsenegative rate is $8 \%$ for $14-\mathrm{G} \mathrm{CB}$ compared with $0.7 \%$ for $\mathrm{VAB}$. There is a risk of underestimating the disease present for lesions of uncertain malignant potential (Cat B3) and suspicious of malignancy (Cat B4) results with $25 \%$ of patients with a B3 biopsy found to have cancer at subsequent surgery and $66 \%$ of those with a B4 biopsy. A $\mathrm{CB}$ diagnosis of in situ malignancy is upgraded to invasive disease at surgery in $15-36 \%$ of patients undergoing $\mathrm{CB}$ and of the order of $10 \%$ with VAB. A high degree of diagnostic accuracy and hence safe patient care can only be achieved by meticulous attention to technique and multidisciplinary cooperation.
\end{abstract}

Keywords Biopsy · Breast · Core needle · Vacuum assisted . Stereotactic

V. Ames $\cdot$ P. D. Britton $(\square)$

Department of Radiology, Cambridge Breast Unit,

Addenbrooke's Hospital,

Box 97, Cambridge, UK CB2 2QQ

e-mail: peter.britton@addenbrookes.nhs.uk

\section{Introduction}

The majority of breast abnormalities are currently biopsied under ultrasound guidance, however certain mammographic microcalcifications and small parenchymal deformities are not demonstrable and thus require stereotactic guidance [1]. Such lesions are almost invariably clinically occult and are demonstrated on mammography. The widespread introduction of national mammographic screening programmes, examining millions of women each year, has led to ever increasing numbers of stereotactic biopsies [2]. The diagnosis and management of these lesions, which are frequently small and of borderline malignant potential, presents the greatest tests of radiological and pathological skill [3]. Improvements in guidance techniques and needle technology have enabled extremely high diagnostic accuracy to be achieved. The high level of concordance between pre-operative and surgical diagnoses is essential to minimize surgical procedures and avoid misdiagnosis [4]. This said, there is still a need for improvement in reducing underestimation of disease and refining patient management of non-malignant high-risk lesions.

In this paper, we aim to compare and contrast the available stereotactic equipment and describe the variety of needle types used, as well as their affect on pathological results and subsequent patient management.

\section{Stereotactic equipment}

Stereotaxis uses co-ordinates defined from oblique radiographs to define accurate needle placement. Initial stereotactic devices were "added-on" to standard mammography 
units using analogue imaging and biopsies were performed with the patient sitting upright. Acquiring target and needle position images were slow, patient movement was common and there was a syncopal rate of approximately $1 \%$ [5]. In the mid 1980s, prone biopsy devices were introduced that were specifically designed for stereotactic biopsy. Patients lay prone during procedures, which improved patient comfort, reduced movement and eradicated syncope. The additional gravitational effect also facilitated the biopsy of lesions close to the chest wall.

The advent of digital imaging dramatically reduced image acquisition time, enabled shorter procedure times and reduced the likelihood for patient movement. Digital technology also allowed post-processing (e.g. black-white image reversal), which may improve calcium visualization [6]. Although the prone biopsy devices have several clear advantages over add-on systems, they cannot be utilized other than for biopsy and are thus expensive and frequently under-utilized. Also, the calcium retrieval rates using 14-G core biopsy (CB) and digital stereotactic equipment are similar at $86 \%$ for both prone [7] and upright systems [6]. There is, however, an appreciable syncopal rate associated with upright biopsy and, although rates as low as $0.3 \%$ have been reported, others have described syncope in up to $35 \%$ of patients [8].

During the late 1990s, the add-on equipment was adapted to enable the procedure to be performed in the decubitus position [9]. Such systems combined some advantages of the prone table, such as reduced syncope, with the ability to perform standard mammography. Although there is a relative paucity of published data on the utility of these devices, the advantage of being able to perform biopsies with the patient lying down and to undertake standard mammography at other times makes such devices both cost and space efficient. More recently, Giotto (manufactured by IMS) have marketed a device which is capable of performing stereotactic biopsies with the patient in a prone, upright or lateral decubitus position as well as undertaking standard digital mammography.

\section{Choice of needle}

Initial stereotactic biopsy utilized fine-needle aspiration cytology (FNAC). Although impressive results could be obtained, it required a dedicated team of experts, particularly for cytopathological interpretation [10], and could not be reproduced when FNAC was introduced on a large scale as in the UK screening programme [11]. The small size and frequent paucicellularity of lesions biopsied under stereotaxis, as well as the lack of reliably identifying calcification in the biopsy specimen, has meant that FNAC is now discouraged from use during stereotactic biopsy [12].
Image-guided large-gauge CB was first reported in 1990 [13-16] and was fairly quickly established as the biopsy technique of choice for the majority of breast lesions. $\mathrm{CB}$ outperforms FNAC on almost all parameters of diagnostic accuracy [17]. Long-throw 14-G needles produce specimens of approximately $30 \mathrm{mg}$ and outperform smaller gauges $(18 \mathrm{G}, 16 \mathrm{G})$ of $\mathrm{CB}$. When sampling microcalcifications, all $\mathrm{CB}$ specimens must undergo radiography to confirm that representative calcification has been sampled. Bagnall et al. [18] recommended that at least three flecks of calcification be seen in at least two cores and preferably five flecks or more should be seen in three cores. Diagnostic accuracy will depend upon the proportion of a cluster of microcalcification that is sampled and, as $\mathrm{CB}$ rarely removed an entire cluster, this led to the development of biopsy devices using vacuum assistance to deliver ever larger volumes of tissue. The vacuum-assisted biopsy (VAB) method uses larger gauge probes (11-7 G) than CB delivering 100-300 $\mathrm{mg}$ of tissue per sample. It incorporates a vacuum chamber to draw tissue into the cutting needle, where the sample is then taken. This technique uses just one puncture, with the probe of the device remaining within the breast, at the site of interest, throughout the sampling [19].

There are a variety of available VAB devices, each with different strengths and weaknesses. The first VAB device marketed was from Mammotome, now a division of Devicor Medical Products. They currently produce both 8$\mathrm{G}$ and $11-\mathrm{G}$ needles. The $8-\mathrm{G}$ needle has a blade and is thus advocated for deployment through dense breast tissue. Mammotome products also have a variable aperture sleeve for use in thin breasts and superficial lesions. The individual specimens need to be removed from the biopsy chamber by hand and the position of the biopsy chamber is controlled manually. Suros Surgical Systems, a Hologic company, produce two levels of product for their second generation of VAB. ATEC is the standard device that uses $9-G$ and $12-G$ needles. It is a closed system that automatically and continuously irrigates the biopsy cavity. In addition, the device can be programmed to continuously acquire specimens every $4.5 \mathrm{~s}$, from pre-programmed positions in the breast. Eviva is the more advanced system from Hologic that incorporates a patent-pending Y-valve for more efficient analgesic application. In addition, there is better visualization of the specimen cores via an in-built tocar tip for better tissue penetration. The EnCor Breast Biopsy System, manufactured by SenoRX, a newly incorporated division of Bard Biopsy Systems, is a second-generation VAB. There is a wide variety of needle sizes available: $7 \mathrm{G}, 10 \mathrm{G}$ and $12 \mathrm{G}$, and there is a halfsampling option for thin breast or 'difficult to access' lesions. The EnCor 360 is an automatic sampling device, with the probe sampling sweep pre-programmed. The 
samples are delivered into a collection chamber. The Vacora Breast Biopsy System is manufactured by Bard Biopsy Systems. The device supports both $10-\mathrm{G}$ and $14-\mathrm{G}$ needles, and there are long-probe and larger sampling chamber options. Although more cost-efficient, the probe needs to be removed following each specimen core acquisition to retrieve the core sample. Although the manufacturer has produced a radiolucent coaxial cannula to aid this, the multiple entries inevitably result in slower delivery of specimens.

The larger sample volumes produced by VAB devices allow more extensive sampling and even complete removal of some clusters of microcalcifications. Kettritz et al. [20] in a trial involving 2,874 patients in five centres using 11-G VAB removed $76 \%$ of clusters measuring less than $10 \mathrm{~mm}$ and even removed $30 \%$ of clusters measuring $11-20 \mathrm{~mm}$ in diameter. Given this likelihood of complete removal of the mammographic abnormality, it is now routine practice to deploy a magnetic resonance imaging (MRI)-compatible localization clip at the time of biopsy. Greater sampling yield, however, is accompanied by a 20 -fold increase in price over 14-G CB. Advocates of VAB, however, would argue that this price increase is offset by a reduced need for diagnostic surgical excision.

More recently, there has been interest in the utilization of larger-gauge needles at vacuum biopsy. However, the evidence to date suggests that there is no significant difference in pathological upgrade rates using a 9-G over an 11-G needle [21]. In addition, a separate study demonstrated that the only significant advantage that an 8-G needle had over an $11-\mathrm{G}$ needle was in the context of firm breast tissue, where the presence of the bladed tip aided accurate needle positioning. Importantly, there was also no statistically significant difference in the complication rates or their severity when using the larger needle gauge. However, the larger-gauge needles are generally more expensive and so their routine use has cost implications [22].

We will explore the differences in diagnostic accuracy between VAB and $\mathrm{CB}$ by examining the histopathological results that one might expect with each and discuss their significance for patient management.

\section{Performance of the stereotactic procedure}

Patient amenities should be a priority, as allaying anxiety prior to the procedure is crucial to patient comfort and compliance, and eventual successful outcome. Good initial preparation is paramount and the radiologist should carefully plan the route of needle placement that optimizes patient comfort, utilizing theshortest needle track within the breast without causing damage to the skin. Following careful patient positioning, meticulous targeting of the lesion using the scout view and stereotactic paired images ensures accurate needle placement. Once the local anaesthetic is instilled, and the needle has been placed to the designated position, a further pair of stereo images is obtained to ensure good needle placement. The tissue samples are then obtained. After 12-24 cores have been collected, a specimen radiograph is performed to ensure that adequate microcalcification has been retrieved. Once this is confirmed, a localization clip is inserted via the biopsy needle, and deployed into the biopsy cavity. Post-clip stereotactic films are then taken to ensure accurate clip deployment, and the entire biopsy system is removed [23]. Application of pressure locally, following the procedure, aids to minimize haematoma formation.

\section{Pathology results}

European guidelines stipulate that all breast needle biopsies should be classified to B1-B5 categories according to histopathological appearances (Table 1) [3]. Each biopsy should be discussed at a multi-disciplinary team meeting, where both pathological and radiological information can be used to decide on the best and safest patient management.

\section{Category B1: normal tissue}

If no calcium is identified on a specimen radiograph and the pathologist only identifies normal breast tissue on a biopsy specimen, then a satisfactory diagnosis has not been reached. Failure to retrieve calcification occurs in $14 \%$ of $\mathrm{CB}$ and $1 \%$ of VAB [7]. A false-negative rate of failed malignancy diagnosis of up to $74 \%$ has been reported when no calcification is evident on the specimen radiograph [18]. Consequently, if no calcium is identified on the radiograph of specimen cores, then the management of choice is either a repeat biopsy or diagnostic excision. Early interval screening should not be considered as a satisfactory alternative patient management [12].

\section{Category B2: benign lesion}

This is the commonest result obtained from a stereotactic biopsy, whether using $\mathrm{CB}$ or $\mathrm{VAB}$, and is obtained between 66 and $75 \%$ of biopsies. If a cluster of calcification has been adequately sampled with extensive amounts of calcium on the specimen radiograph and the pathologist finds benign changes associated with visible calcification, then this is a very safe diagnosis and the patient can be reliably reassured and discharged without further intervention [3]. When faced with a B1 or B2 biopsy result, the concern to the breast radiologist is whether malignancy 
Table 1 Categories of pathological interpretation of large-gauge core or vacuum-assisted breast biopsy as specified in the European guidelines for quality assurance in breast cancer screening and diagnosis [3]

\begin{tabular}{lll}
\hline Category & Description & Examples \\
\hline B1 & Normal & Normal tissue or uninterpretable \\
B2 & Fenign lesion & Fibrocystic change, \\
& fibroadenoma, \\
& sclerosing adenosis, \\
& duct ectasia, \\
& Lesion of uncertain potential & fat necrosis \\
& & Papillary lesion, \\
B3 & radial scar/complex sclerosing lesion, \\
& & lobular intraepithelial neoplasia, \\
& & atypical ductal hyperplasia, \\
& & phyllodes tumour, \\
& & mucocele-like lesions. \\
B4 & Atypical ductal hyperplasia \\
B5 & Malignant & May be subdivided into malignant-in-situ or malignant invasive \\
\hline
\end{tabular}

could still be present. The likelihood of such a falsenegative result varies depending upon the needle type used. The false-negative rate for $14-\mathrm{G} \mathrm{CB}$ is $8 \%$ compared with $0.67 \%$ for VAB [24]. Assuming the abnormality has been adequately sampled, there is no current evidence to suggest that there is a role for specific follow-up in biopsy-proven benign disease.

Category B3: lesion of uncertain malignant potential, and Category B4: suspicious of malignancy

These categories mainly consist of lesions which may provide benign histology on needle biopsy but are known to either show heterogeneity or have an increased risk of associated malignancy. The B3 category has a lower rate of malignancy on further surgical biopsy (25\%) when compared with B4 (66\%) [3]. The majority of B3 lesions require surgical excision, but all such patients should be discussed at a multidisciplinary meeting. A limitation of non-surgical biopsy that requires consideration is disease underestimation. It is well documented that there is a risk of underestimating the disease profile for B3/4 results, i.e. when a lesion is initially diagnosed as atypical ductal hyperplasia $(\mathrm{ADH})$ at biopsy and is subsequently upgraded to ductal cancer in situ (DCIS) following surgery. The rate of underestimation of ADH to DCIS is between 20 and $56 \%$ for $\mathrm{CB}$ and $19 \%$ for $\mathrm{VAB}$ [25-28]. The main pathological difference between $\mathrm{ADH}$ and DCIS is the amount of involved tissue, and that atypical epithelial proliferation can be categorized as either B3 or B4, dependent on severity and extent [29]. It therefore follows that if sampled tissue volume is maximized using VAB, then the incidence of disease underestimation will be minimized. Although there is much debate whether there is a subset of B3 lesions that might not warrant surgical excision because their upgrade rate is so low there is, as yet, no reliable consensus. In addition, all research undertaken so far has indicated that complete removal of microcalcification at VAB should not be considered therapeutic as residual tumour is often still present at surgery. However, there is evidence that total removal of the index microcalcification does correlate with decreased pathological upgrade rates at surgery [30]. It is as yet unclear which histopathological needle biopsy findings, although atypical, are of sufficiently low risk that surgical excision is not warranted. This balance between safe clinical practice and overtreatment is contentious and a unified view has yet to emerge. As a result, at present, the NHSBSP guidelines advise surgical diagnostic excision of all B4 lesions and the management of all B3 lesions should be discsussed at an multi-disciplinary team meeting [29]. There is no current evidence to suggest further imaging is of benefit when a representative tissue sample has been obtained.

\section{Category B5: malignant}

These can be subdivided into malignant in situ (Cat: B5a) or malignant invasive (B5b) [29]. When sampling microcalcification, DCIS is the commonest malignancy diagnosed and constitutes $20 \%$ of screen detected cancer [2, 4]. As with B3/B4 lesions, underestimation of disease is possible where a B5a (in situ malignancy) is upgraded to invasive malignancy following surgery. The underestimation rate is again dependent upon the needle type used. A $\mathrm{CB}$ diagnosis of DCIS is up-graded to invasive disease at surgery in $15-36 \%$ of patients, whereas of the order of $10 \%$ 
with VAB [31]. The factors associated with an increased likelihood of upgrade to invasive disease are the number of flecks of microcalcification, the size of the cluster and the grade of the DCIS. Clusters that contain more than 40 pieces of calcification have a $48 \%$ chance of invasion at final histology compared with $15 \%$ for clusters with less than 40 pieces of calcification. Clusters that are less than $11 \mathrm{~mm}$ in diameter have an $18 \%$ chance of invasion at final histology compared with $35 \%$ for clusters $60 \mathrm{~mm}$ or more in diameter [32]. Thirteen percent of patients initially diagnosed with low grade DCIS will be upgraded to invasive disease, compared with $36 \%$ of those with highgrade DCIS [33]. This has important consequences for patient management. As the majority of patients with invasive disease will require surgical axillary staging, surgeons not infrequently perform axillary surgery in patients with biopsy-proven DCIS if upgrade to invasion is thought likely at surgery. As a consequence, data from the UK show that $31 \%$ of patients with a final diagnosis of DCIS only will also have had their nodal status ascertained at the time of breast surgery [4]. The vast majority of these patients will have undergone mastectomy, however $10 \%$ of patients undergoing conservation therapy for DCIS had also undergone axillary staging. Although this will almost invariably be in the form of sentinel lymph node biopsy, of proven low morbidity, it nonetheless constitutes an unnecessary surgical procedure.

The importance of accurate non-operative diagnosis cannot be underestimated. It enables the safe discharge of those patients with benign disease and the planning of correct surgery for those with malignancy [34].

\section{Complications}

The complication rates of stereotactically guided needle biopsy are surprisingly low, and any adverse events are usually minor. Reported complications include bruising, discomfort, haematoma and abscess formation. The rate of requirement for surgical drainage of an abscess or haematoma is in the region of $0.1 \%$ [19]. Seeding of tumour cells has been reported but does not appear to be of clinical significance [35].

\section{Conclusions}

The sensitivity and specificity of stereotactically guided largecore-needle breast biopsy is high, even for small mammographic distortions and microcalcifications. Vacuum-assisted biopsy systems consistently out-perform 14-G CB, with lower disease underestimation and false-negative rates, and the reduced need for diagnostic or multi-treatment surgery offsets the considerable added procedural expense. Although a high degree of diagnostic accuracy is achievable, even on a national scale $(84 \%$ of the 3,491 patients with screendetected DCIS were diagnosed pre-operatively in the UK screening programme in 2008-2009) greater efforts are required to minimize the false-negative rate and the underestimation of DCIS and invasive malignancy [4]. It is only by meticulous attention to technique and a high degree of multi-disciplinary cooperation that diagnostic accuracy will increase with consequent improvement in patient care.

Acknowledgements Dr Peter D. Britton is supported with research funding from the National Institute for Health Research (NIHR) Cambridge Biomedical Research Centre (BMRC).

\section{References}

1. Wallis M et al (2007) Guidelines from the European Society of Breast Imaging for diagnostic interventional breast procedures. Eur Radiol 17:581-588

2. NHS Breast Screening Programme (2009) NHS breast screening programme review ISBN 978184463064 6. Available via www. cancerscreening.nhs.uk//breastscreen/publications/2009review. html Accessed 21 August 2010

3. Perry $\mathrm{N}$ et al (2006) European guidelines for quality assurance in breast cancer screening and diagnosis, 4th edn, ISBN 92-7901258-4. Available via www.euref.org/ND7306954ENC_002.pdf Accessed 21 August 2010

4. NHS Breast Screening Programme \& Association of Breast Surgery at BASO (2010) An audit of screen-detected breast cancers for the year of screening April 2008 to March 2009. http:// www.cancerscreening.nhs.uk/breastscreen/publications/baso20082009.pdf Accessed 21 August 2010

5. Kirshenbaum KJ et al (2003) Stereotactic core needle biopsy of nonpalpable breast lesions using a conventional mammography unit with an add-on device. AJR Am J Roentgenol 181:527-531

6. Whitlock JP et al (2000) Digital imaging improves upright stereotactic core biopsy of mammographic microcalcifications. Clin Radiol 55:374-377

7. Jackman RJ et al (2006) Breast microcalcifications: retrieval failure at prone stereotactic core and vacuum breast biopsyfrequency, causes, and outcome. Radiology 239:61-70

8. James $\mathrm{J}$ et al (2005) The use of a short-acting benzodiazepine to reduce the risk of syncopal episodes during upright stereotactic breast biopsy. Clin Radiol 60:394-396

9. Doyle AJ et al (1999) Decubitus stereotactic core biopsy of the breast: technique and experience. AJR Am J Roentgenol 172:688 690

10. Azavedo E et al (1989) Stereotactic fine-needle biopsy in 2594 mammographically detected non-palpable lesions. Lancet 8646:1033-1036. doi:10.1016/S0140-6736(89)92441-0

11. Britton PD et al (1999) Needle biopsy in the NHS breast screening programme 96/97: how much and how accurate? Breast 8:5-11

12. NHS Breast Screening Programme (2010) Clinical guidelines for breast cancer screening assessment, 3rd edn, ISBN 978-1-84463068-04. Available via www.cancerscreening.nhs.uk/breastscreen/ publications/nhsbsp49.html Accessed 21 August 2010

13. Parker SH et al (1990) Stereotactic breast biopsy with a biopsy gun. Radiology 176:741-747

14. Parker SH et al (1993) US-guided automated large-core breast biopsy. Radiology 187:507-511 
15. Parker SH et al (1994) Percutaneous large core breast biopsy. Cancer 74:256-262

16. Parker SH et al (1994) Percutaneous large-core breast biopsy: a multi-institutional study. Radiology 193:359-364

17. Britton PD (1999) Fine needle aspiration or core biopsy. Breast 8:1-4

18. Bagnall MJ et al (2000) When have mammographic calcifications been adequately sampled at needle core biopsy? Clin Radiol 55:548-553

19. Burbank F et al (1996) Stereotactic breast biopsy: improved tissue harvesting with the Mammotome. Am Surg 62:738-744

20. Kettritz U (2004) Stereotactic vacuum-assisted breast biopsy in 2874 patients: a multicenter study. Cancer 100:245-251

21. Laurenco AP et al (2007) Stereotactic breast biopsy: comparison of histologic underestimation rates with 11- and 9-gauge vacuumassisted breast biopsy. AJR Am J Roentgenol 189:W275-W279

22. Hahn M et al (2008) Vacuum assisted breast biopsy: a comparison of 11-gauge and 8-gauge needles in benign breast disease. World $\mathrm{J}$ Surg Oncol 6:51

23. Parker $\mathrm{S}$ et al (1997) Performing a breast biopsy with a directional, vacuum-assisted biopsy instrument. Radiographics $17: 1233-1252$

24. Jackman RJ et al (2009) False-negative diagnoses at stereotactic vacuum-assisted needle breast biopsy: long-term follow-up of 1,280 lesions and review of the literature. AJR Am J Roentgenol 192:341-351

25. Darling ML et al (2000) Atypical ductal hyperplasia and ductal carcinoma in situ as revealed by large-core needle breast biopsy: results of surgical excision. AJR Am J Roentgenol 175:1341-1346

26. Meyer JE et al (1998) Large-needle core biopsy: nonmalignant breast abnormalities evaluated with surgical excision or repeat core biopsy. Radiology 206:717-720
27. Philpotts LE et al (1999) Comparison of rebiopsy rates after stereotactic core needle biopsy of the breast with 11-gauge vacuum suction probe versus 14-gauge needle and automatic gun. AJR Am J Roentgenol 172:683-687

28. Houssami $\mathrm{N}$ et al (2007) Borderline breast core needle histology: predictive values for malignancy in lesions of uncertain malignant potential (B3). Br J Cancer 96:1253-1257

29. NHS Breast Screening Programme (2001) NHSBSP 50: guidelines for non- operative diagnostic procedures and reporting in breast cancer screening. isbn 187199744 5. Available via http:// www.cancerscreening.nhs.uk/breastscreen/publications/pathology. html\#p-pr Accessed 22 August 2010

30. Penco S et al (2010) Stereotactic vacuum-assisted breast biopsy is not a therapeutic procedure even when all mammographically found microcalcifications are removed: analysis of 4,086 procedures. AJR Am J Roentgenol 195:1255-1260

31. Jackman RJ et al (2001) Stereotactic breast biopsy of nonpalpable lesions: determinants of ductal carcinoma in situ underestimation rates. Radiology 218:497-502

32. Bagnall MJ (2001) Predicting invasion in mammographically detected microcalcification. Clin Radiol 56:828-832

33. O'Flynn EA et al (2009) Prediction of the presence of invasive disease from the measurement of extent of malignant microcalcification on mammography and ductal carcinoma in situ grade at core biopsy. Clin Radiol 2009(64):178-183

34. Wallis MG et al (2009) Non-operative diagnosis-effect on repeat-operation rates in the UK breast screening programme. Eur Radiol 19:318-323

35. Liberman L et al (1999) Epithelial displacement after stereotactic 11-gauge directional vacuum-assisted breast biopsy. AJR Am J Roentgenol 172:677-681 